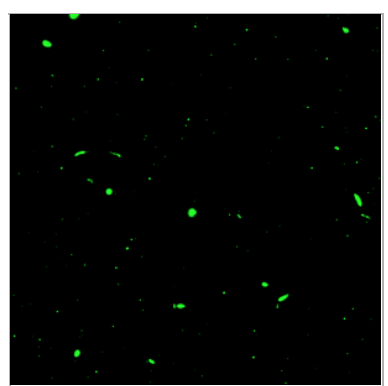

\section{VERSION 5}

JUN 06, 2018

\section{OPEN OACCESS}

DOI:

dx.doi.org/10.17504/protocol s.io.qsgdwbw

\section{External link:}

https://doi.org/10.1371/journ al.pone.0184371

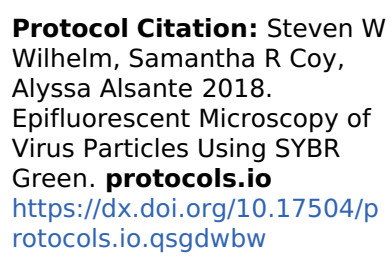

\section{MANUSCRIPT CITATION:}

Gainer PJ, Pound HL, Larkin

$A A$, LeCleir GR, DeBruyn JM, Zinser ER, Johnson ZI, Wilhelm SW (2017)

Contrasting seasonal drivers

of virus abundance and production in the North Pacific Ocean. PLoS ONE 12(9): e0184371. doi: 10.1371/journal.pone.018437 $\underline{1}$

License: This is an open access protocol distributed under the terms of

the Creative Commons

Attribution License, which permits unrestricted use,

distribution, and reproduction in any medium, provided the original author and source are credited

Protocol status: Working We use this protocol and it's working

Created: Jun 06, 2018

Last Modified: Jun 06, 2018

\section{(3) Epifluorescent Microscopy of Virus Particles Using SYBR Green V.5}

\section{Steven W Wilhelm ${ }^{1}, \quad$ Samantha R Coy ${ }^{1}$, Alyssa Alsante ${ }^{1}$}

${ }^{1}$ The University of Tennessee, Knoxville

The Aquatic Microbial Ecology Research Group - AMERG (The Buchan, Zinser and Wilhelm labs)
Ashley A Humphrey

University of Tennessee, Knoxville

\title{
ABSTRACT
}

Adapted from: Ortmann and Suttle (2009) Determination of Virus Abundance by Epifluorescence microscopy. Ch. 10 Methods of Molecular Biology.

Contact Dr. Steven Wilhelm (wilhelm@utk.edu) or Samantha Coy (srose16@vols.utk.edu) for additional information regarding this protocol.

\section{ATTACHMENTS}

Epifluorescent

Microscopy.pdf

\section{PROTOCOL integer ID:} 12840 


\section{Solutions}

Make a 1\% p-phenylenediamine (antifade) solution - $0.01 \mathrm{~g}+1 \mathrm{~mL}$ Milli-Q water.

Vortex, keep in the dark.

3

Retrieve an 8 uL stock of 1,000x SYBR Green, DI water, and 225 uL 50:50 PBS/Glycerol solutions

Add 6 uL SYBR Green to 235 uL filtered DI. Keep in the dark.

5 Add $25 \mathrm{uL}$ 1\% p-phenylenediamine to the $225 \mathrm{uL}$ aliquot of 50:50 PBS/Glycerol. Keep in the dark.

6 Place the SYBR Green and antifade solutions into a drawer while working.

Note

Only the red light can be on when the solutions are out of the drawer.

\section{Sample Preparation}

7 Fix sample with $0.5 x$ glutaraldehyde.

Note

If you are going to freeze your sample between slide preparation and visualization, then you need to cool the fixed sample in the dark at $4^{\circ} \mathrm{C}$ for $15-30 \mathrm{~min}$, and flash freeze in liquid nitrogen before storing at $-80^{\circ} \mathrm{C}$.

\section{Vacuum Setup}

8 Turn on the vacuum (no more than $\sim 25 \mathrm{~mm} \mathrm{Hg}$ ).

9 Rinse wells with Milli-Q water. 
10

While the knobs are turned to open (vacuum on), use the tweezers to add the 0.45 um nitrocellulose backing filter.

Note

This filter is used to protect the more fragile 0.02 um Anodisc filter that the viruses are collected on.

11 Once the filter is on, you should add a few drops of Milli-Q and then turn the knobs to closed (vacuum off).

12

Use tweezers to apply the 0.02 um Anodisc filter to the top of the backing filter.

13 Turn the vacuum back on by turning the knobs to open and add the sample in two 500 uL aliquots.

\section{SYBR Green}

14 Pipette SYBR Green as dots onto a glass petri dish. Make enough dots for each filter.

\section{\ $30 \mu \mathrm{L}$}

15 With the vacuum on, use a needle and tweezers to remove the Anodisc filter and place onto a dot. Turn the vacuum off.

16 Put the lid over the plate and back into the drawer to stain for $20 \mathrm{~min}$.

\section{(3) 00:20:00}

\section{Slide Preparation}

17 During the wait, label slides with sample ID, dilution, stain, sample volume, and date.

$$
\text { Note }
$$

You may turn the white light on during this period switching it off anytime the stain is exposed to light.

18 Re-apply Milli-Q to the backing filter and place the stained Anodisc on top. Add another drop of Milli-Q. 
19 Turn the vacuum on again. After the stain has been washed away, use the needles and tweezers to remove the Anodisc--again, with the vacuum still on.

20 Place the filter onto a Kim Wipe to dry.

21 On the labeled side, add $18 \mathrm{uL}$ antifade solution, apply the dried Anodisc filter and apply another 18 uL of antifade to the top.

22 Apply a coverslip and lightly press down so there are no bubbles.

\section{Storage}

23 Store at $-20^{\circ} \mathrm{C}$ in the dark for up to four months (3-4 weeks recommended). .

Note

Thawing the sample repeatedly will result in fading of the stain, but you can attempt to re-stain the filter if it fades

\section{Microscope}

24 Turn on all three computers and the shutter.

25 Open the LAS AF program.

26 On the second menu, use the FLUO--Filter cubes L5 and the FLUO incident settings.

27 On the fourth menu, click set/clear focus position to desired bottom using the up and down arrows. Once positioned, click "set". You can then move the microscope slide to the desired position to visualize the slide and click "set". These settings will then be saved within the microscope.

*Z: up/down (turn the back knob) 
*X/Y: Side to side (turn the top or bottom half of the front knob)

28 Once the options are set and the microscope slide is set into place, you can then turn the shutter on/off on the first menu--'IL-Shutter ON/OFF'.

29 Rod all of the way in on the microscope -- image only on the micrscope itself Rod half way in -- image on the micrscope and the computer screen Rod all of the way out -- image only on the computer screen

\section{Computer Imaging Software}

30 Under the acquire --> acquisition tab:

*To look at a preview of your image, click "live" (delay may occure due to exposure)

*Set desired exposure, gain and intensity.

*To save a desired image, click "capture image". The image will appear on the right screen, where you can adjust the background fluorescence by moving the bar on the black to green scale at the bottom lefthand corner.

31 Under the acquire --> experiment tab:

*The captured images will automatically save to the experiment .lif file. This can only be viewed using this software.

*Right clock on an image to rename/delete it.

*Click "save all" to save images to the desired directory.

*To save images in a different format, right click, select export, and chose your desired format (e.g. JPEG, TIFF, or the format requried for your analysis software).

\section{Enumeration of Virus-Like Particles}

32 With the rod all of the way in, use the fine adjustment knob to locate and resolve the viruses into focus.

33 Count all of the viruses in the grid. The ideal concentration is between $20-40$ viruses per grid so that you have enough time to count the viruses before they fade.

34 After counting all of the viruses in the field, look up from the microscope, and move the stage to a different part of the slide to count viruses in another field. It is imprtant to do this without looking so that you can randomly sample your filter and not incur a bias in your counts.

35 Repeat this process until you have counted enough fields to total between 200-300 viruses. Calculate the average, and determine the $\mathrm{VLP} / \mathrm{mL}$ by the equation:

$($ Avg VLP/field $) \star(28352.9) \star($ Dil. factor $) \star(1000 /$ volume filtered $(u L)) \star((1000+$ glu. volume $(u L)) / 1000)$ 